\title{
Proton CT Setup at CV-28 of IEN/CNEN
}

\author{
I. Evseev ${ }^{1}$, J. Teixeira de Assis ${ }^{1}$, O. Yevseyeva ${ }^{1}$, U.M. Vinagre Filho ${ }^{2}$, J.A.P. Setti ${ }^{3}$, \\ H.R. Schelin ${ }^{3}$, S.A. Paschuk ${ }^{3}$, M.C.L. Klock ${ }^{3,4}$, R.W. Schulte ${ }^{4}$, and R.T. Lopes ${ }^{5}$ \\ ${ }^{1}$ Polytechnic Institute of UERJ - Nova Friburgo, RJ, Brazil \\ ${ }^{2}$ Institute of Nucler Engineering - CNEN, Rio de Janeiro, RJ, Brazil \\ ${ }^{3}$ Federal Center of Technological Education - CEFET/PR, \\ Av. Sete de Setembro, 3165, CEP 80230-901, Curitiba, PR, Brazil \\ ${ }^{4}$ Loma Linda University Medical Center, Loma Linda - CA - USA and \\ ${ }^{5}$ Nuclear Instruments Laboratory - COPPE/UFRJ, Rio de Janeiro, RJ, Brazil
}

Received on 21 December, 2004

\begin{abstract}
Although the idea of proton Computed Tomography (pCT) is not new, it is currently not available. It is still unknown if the image quality of pCT is sufficient for localizing tumors and if the accuracy of proton range calculations surpass that of X-ray CT based range calculations without significantly increasing the patient exposure. The spatial resolution of $\mathrm{pCT}$ is limited mainly by multiple Coulomb scattering, while the contrast resolution is affected principally by the statistical nature of the proton energy loss mechanism. Both effects are more pronounced and thus easier to study at the low proton energies available at CV-28. An experimental setup for pCT at CV-28 was elaborated by our team. The first-generation CT scheme was assumed as the most adequate for the existing CV-28 proton "pencil" beam. All equipment, which includes the collimators for the initial and outgoing proton beam, the tomographic turntable, and the ORTEC L-035-025-5 proton detector, was assembled onto the support bar of the ORTEC vacuum chamber. A water-filled glass tube of $5 \mathrm{~mm}$ external diameter with a $1 \mathrm{~mm}$ diameter polyethylene rod on the central axis was chosen as the first sample to be studied. Later, the water will be substituted by tissue-equivalent solutions of different densities to study the contrast resolution. A Monte Carlo simulation of the experiment, assuming $25 \mathrm{MeV}$ monochromatic proton beam, was made using the SRIM-2003.20 code. The simulation permitted to choose the critical parameters for the setup, such as the beam collimation, the steps of translation and rotation of the turntable, and the required statistics for an accurate measurement of proton energy.
\end{abstract}

\section{INTRODUCTION}

Proton radiation therapy is a highly precise form of cancer treatment, which spares more healthy tissue and allows higher tumor doses than conventional radiation therapy [1]. In existing centers, proton treatment is planned using conventional $\mathrm{X}$-ray computed tomography (CT) data [2]. This method has limited accuracy because the information about X-ray absorption across the patient's body does not reflect all physical parameters needed to calculate the proton range and depth dose distribution [3].

Alternatively, the proton treatment gantry could be used to scan the patient and to reconstruct the electron density distribution needed for proton treatment planning from measured proton energy loss data [4]. The energy of protons produced by the synchrotron at Loma Linda University Medical Center [5] and in several other proton treatment centers [6] is sufficiently high for this purpose [7].

Although the idea of proton CT was first described more than 30 years ago, proton CT (pCT) is currently not available. There are yet unanswered questions, such as whether the density resolution of $\mathrm{pCT}$ will be sufficient for accurate proton treatment planning at an acceptable dose to the patient [8]. Another important problem relates to the relationship between spatial resolution of pCT and multiple Coulomb scattering. Therefore, it is essential that additional experimental and theoretical studies be performed.

Some of the unanswered questions can be well studied at the low proton energies, available at the CV-28 Cyclotron of
IEN/CNEN [9]. At the CV-28 cyclotron, protons can be accelerated with variable energy up to $24 \mathrm{MeV}$. This energy is still high enough to pass a proton beam through a relatively thick sample, but at the same time it is low enough to be measured with high precision by completely stopping them in a semiconductor detector [10]. Consequently, the shape of final proton energy spectra after traversing the object can be compared with various analytical calculations [11] and an adequate description of both inelastic and elastic proton scattering can be chosen. This will provide useful information of the physical limitations of pCT with respect to spatial and contrast resolution beyond what is currently known, and will support the development of an adequate method for the pCT image reconstruction.

\section{EXPERIMENTAL SETUP}

The experimental setup represents a miniaturized first generation CT scanner[2], and is located in vacuum (Fig. 1). Thin tubes filled with tissue-equivalent solutions serve as test objects during the initial phase. By varying the solution density in small steps, the contrast resolution of the pCT method would be evaluated. The sample was fixed onto the tomographic turntable, which may be rotated and translated in the direction perpendicular to the proton beam axis. The collimated protons from $\mathrm{CV}-28$ would form a pencil beam of suitable cross section, and registered by the $\mathrm{Si}(\mathrm{Li})$ ORTEC L-035-0255 detector [10], situated behind the turntable. The variable diameter collimator would be installed between the turntable 
and the detector. All these components have to be fixed onto the support of the existing scattering chamber.

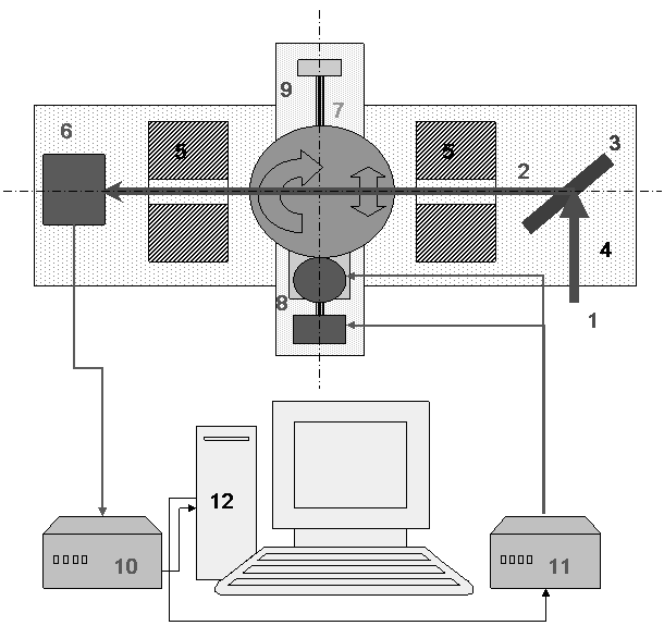

FIG.1: The planned experimental layout: the initial proton beam from CV-28 should be reduced in intensity to an appropriate level (2) via elastic scattering within a target foil (3); the support bar (4), would be used to mount two collimators (5), proton detector (6) and tomographic turntable (7); two step-motors (8) will furnish the turntable with rotation and translation along the motion guide (9); the signal from the detector will be acquired and modified by readout electronics (10), and the mechanical motions should be managed by electronic controls (11) under the supervision of a computer code (12).

\section{MONTE CARLO SIMULATIONS}

A simple computer simulation of the setup performance was done to explore the parameters of the experimental procedure. A monochromatic $25 \mathrm{MeV}$ proton beam passing through a water-filled glass tube of $5 \mathrm{~mm}$ external and $4 \mathrm{~mm}$ internal diameter, with a $1 \mathrm{~mm}$ diameter polyethylene rod on the central axis, was simulated using the SRIM-2003.20 computer code [12]. Due to the peculiar properties of the code, the tube had to be treated as a set of glass, water and polyethylene layers with variable thickness depending on the particular geometry at each projection point.

The length of the parallel projection, i.e., the turntable translation range, was assumed to be $10 \mathrm{~mm}$. For the simulation, 104 proton tracks were generated for each of 25 and 100 equidistant turntable positions on the translation range. Both the energy and position of the outgoing protons were evaluated in the detector plane, situated $10 \mathrm{~cm}$ behind the turntable. The energy values of these 25 and 100 parallel projection points were defined as the average outgoing proton energy for a given detector collimation. The parallel projections were simulated for equidistant turntable angular positions with the angular step, determined as the smallest integer greater than or equal to the value predicted by the Nyquist's theorem [2].

The pCT images were reconstructed using the standard $\mathrm{CT}$ filtered back projection (FBP) procedure [2]. The pro- ton energy values at each projection point were translated into water-equivalent path length, i.e. the thickness of a hypothetical water layer providing the same proton energy loss as the simulated sample along the given trajectory. These values were used as FBP input to reconstruct the relative electron density with respect to water over the sample cross section.

\section{SIMULATION RESULTS}

The glass tube wall and the enclosed water filling are clearly separated on the images of the phantom cross section for both 25 and 100 parallel projections (Fig. 2 and Fig. 3). On the other hand, the polyethylene rod, which has only $6 \%$ less density than water, is visible only on Fig.3. The mathematical features of Fast Fourier Transform algorithm, which is used in the filtered back projection procedure, introduce some small regional oscillations on reconstructed images near the boundaries, enhancing the materials with different densities. This effect becomes important for the low-resolution images; i.e., the one obtained from 25 point projections (see Fig.2).

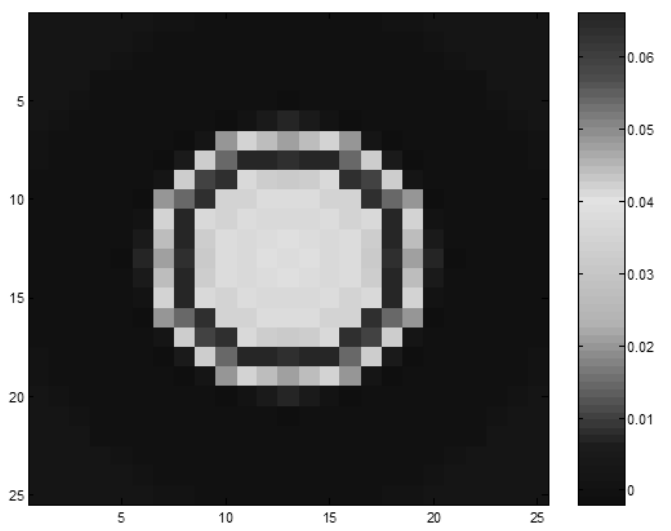

FIG.2: The pCT image reconstructed from 25 projection points, simulated by SRIM-2003.20. The projections were simulated at 45 equidistant angular positions with $4^{\circ}$ steps. The initial number of protons for each projection point was $10^{4}$.

The statistical limitations for pCT image contrast resolution at a given dose will be important. For our simulation, there are no visible statistical fluctuations in the reconstructed images. The number of simulated proton tracks (104 events for each projection point) was obviously high enough to obtain the images with a large signal-to-noise ratio. From the experimental point of view, this number is quite reasonable: For a data-acquisition period of 60 seconds it will correspond to a proton beam current of only $I=2.7 \cdot 10^{-8} n A$. It is an open question whether it will be possible to reduce the beam current to low levels only by a strict collimation, or a beam scattered by a thin foil beam has to be used to achieve small fluences. Therefore, the last option has been included in our experimental setup (see Fig. 1). 


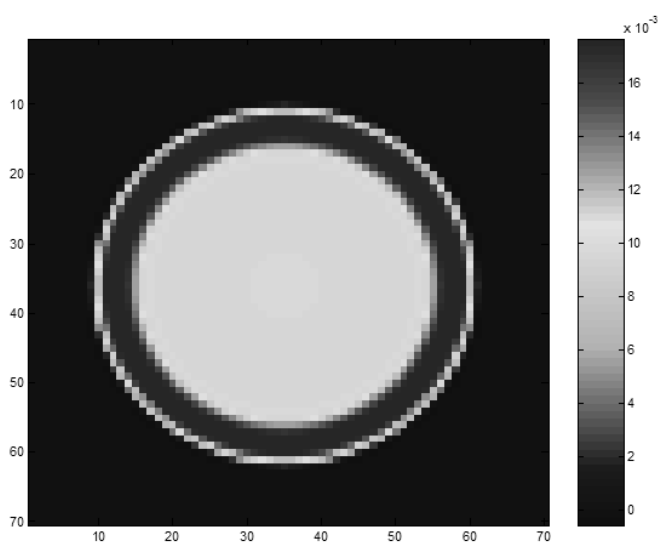

FIG.3: The pCT image reconstructed from 100 projection points. The projections were simulated by SRIM-2003.20 at 180 equidistant angular positions with $1^{o}$ steps. The initial number of protons for each projection point was $10^{4}$.

It should be noted that due to multiple Coulomb scattering, a strict collimation of the detector would significantly reduce the number of protons available for measurement (see Fig. 4). Consequently, decreasing of the collimator aperture is limited not only by the technical difficulty to produce very small holes in arbitrary thick layers, but also by the huge difference in proton beam intensity for the central part and the periphery of the projections.

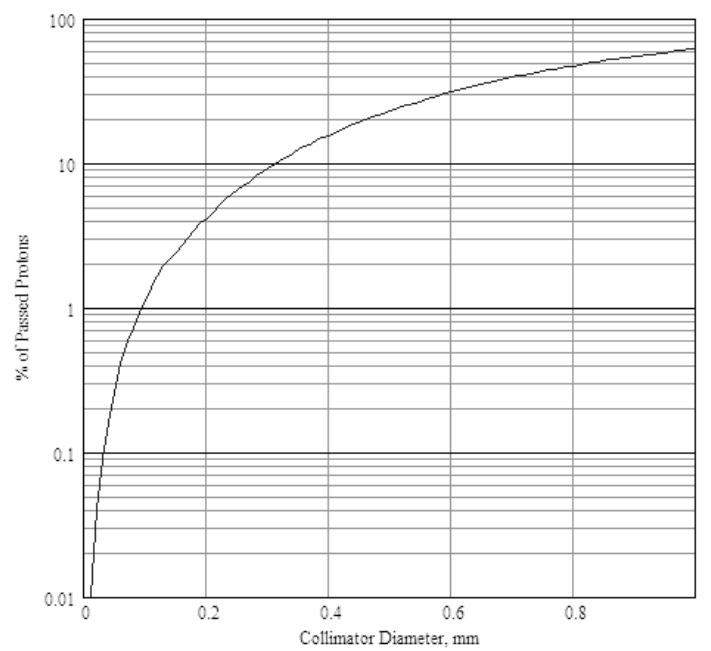

FIG.4: The percentage of protons that would be registered versus the diameter of the collimator in front of the detector. The data were calculated for the central projection point, where the reduction due to multiple Coulomb scattering in the scanned phantom has a maximum.

\section{DISCUSSION}

We have performed a simulation of a proton CT experimental setup using a very simple model, which obviously does not take into account some significant characteristics like the strong asymmetry in proton crossing conditions along the sample boundaries: the degradation of energy resolution due to non-parallel beam collimation, a finite precision of mechanic motion, etc.

Our simulation, however, predicts the general data quality of the intended experiment. The dimensions of the tomographic sample have an upper limit, determined by the mean range of the low-energy protons from the CV-28 accelerator. Another limitation is that the diameter of the collimator aperture in front of detector cannot be made arbitrarily small: In addition to the possible degradation of energy resolution due scattering from the collimator boundaries, there will be a very large difference in proton intensity between projection points and near to and far from the sample center (Fig.4).

Consequently, the main problem was to find a realistic compromise between the desire to have a reasonably high spatial resolution and the given limitations of the CV-28 facility. Based on our simulations, we expect that measurements with rotational steps between $1^{\circ}$ and $4^{\circ}$, translational steps between $0.1 \mathrm{~mm}$ and $0.4 \mathrm{~mm}$, and a collimation between $0.2 \mathrm{~mm}$ and $0.8 \mathrm{~mm}$, should reproduce the main pCT features. These ranges define the engineering requirements for the tomographic turntable.

\section{CONCLUSIONS}

In spite of significant simplifications in our simulations, the results obtained provided a useful guide for the design of the experimental pCT setup at CV-28. At this point, the technical design is complete and all components of pCT system have been manufactured. The pCT system is now in the installation phase at CV-28.

\section{Acknowledgments}

The authors are very thankful to the Brazilian agencies CNPq, ANP and Fundação Araucária for financial support.

R.W. Schulte's research was sponsored by the U.S. Dept of Army (C/A \# DAMD17 - 97-2-7016) and the National Medical Technology Testbed (NMTB), and the content and information does not reflect the position or policy of the U.S. government, or NMTB; no official endorsement should be inferred. 
[1] J. Meikle - How particles can be therapeutic - Physics Web, 2003, Available: http //physicsweb.org/article/world/16/8/9 (Accessed: 2004, July 30).

[2] A.C. Kak and M. Slaney - Principles of Computerized Tomographic Imaging. Society of Industrial and Applied Mathematics (2003). Available: http : //www.slaney.org/pct/ (Accessed: 2004, July 30).

[3] U. Schneider, E. Pedroni, and A. Lomax, Phys. Med. Biol. 41, 111 (1996).

[4] K. M. Hanson, et al., Phys. Med. Biol. 26, 965 (1981).

[5] Proton Treatment Center, 2004, Loma Linda University Adventist Health Sciences Center, Available: http : //www.llu.edu/proton/index.html (Accessed: 2004, July 30).

[6] The Harvard Cyclotron Laboratory, 2001, Harvard University, Available: http : //neurosurgery.mgh.harvard.edu/hcl/ (Accessed: 2004, July 30).

[7] R. W. Schulte, IEEE Transactions on Nuclear Science, 51, 866
(2004).

[8] T. Satogata, et al. (2003), Dose / Sensitivity in Proton Computed Tomography, Brookhaven National Laboratory, Available: http : //www.agsrhichome.bnl.gov/AP/ap ${\text { otes } / \text { ap }_{n} \text { ote }}_{1} 20 . p d f$ (Accessed: 2004, July 30)

[9] Instituto de Engenharia Nuclear, The Brazilian Nuclear Energy Commission, Available: http ://www.ien.gov.br/index.htm (Accessed: 2004, July 30).

[10] L Series Room-Temperature Lithium-Drifted Silicon Detectors, ORTEC, Available: www.ortec online.com/detectors/charged particle/l $l_{s}$ eries.htm (Accessed: 2004, July 30).

[11] V.S. Remizovich, D. B. Rogozkin, and M. I.Ryazanov - Analytical Description of the Fast Particle Penetration in Matter.

[12] Ziegler, J.F. Particle Interactions with Matter, Available: http : //www.srim.org (Accessed: 2004, July 30). 